\title{
Hernias discales calcificadas en la infancia
}

\author{
M. Ortega-Martínez; J.M. Cabezudo; I. Fernández-Portales; L. Gómez-Perals y L.M. Bernal-García
}

Servicio de Neurocirugía. Hospital Regional Universitario Infanta Cristina. Badajoz

\section{Resumen}

Las calcificaciones de los discos intervertebrales son muy infrecuentes en la infancia. Aunque su etiología no está clara, se sabe que no se relacionan con procesos degenerativos. Cursan con clínica inespecífica de raquialgias, contracturas musculares y, a veces, febrícula. Aunque ocasionalmente estos discos se hernian, causando dolor radicular 0 , mucho más raramente, déficits neurológicos o disfagia, la clínica desaparece en la mayor parte de los casos con tratamiento conservador, y sólo excepcionalmente se precisan tratamientos más agresivos. En nuestra revisión de la literatura hemos encontrado solamente 17 casos que requirieron tratamiento quirúrgico. En dos de estos casos el paciente estaba previamente diagnosticado de calcificaciones intervertebrales idiopáticas, pero no se habían descartado hernias preexistentes mediante resonancia magnética (RM). Presentamos un nuevo caso de una paciente diagnosticada a la edad de 10 años de calcificaciones discales idiopáticas, sin hernias discales en la RM, en la que 4 años después uno de los discos cervicales calcificados se hernió presentando un cuadro de radiculopatía cervical con déficit motor, precisando discectomía y artrodesis intersomática. Consideramos que este caso prueba definitivamente la teoría de Heinrich y cols., que considera la hernia calcificada en la infancia como la complicación de una patología previa, la calcificación del disco intervertebral. Se discuten los aspectos clínicos y terapéuticos de esta entidad.

PALABRAS CLAVE: Calcificación del disco intervertebral. Discectomía cervical. Hernia de disco calcificada. Hernia de disco en la infancia.

Calcified disc herniation in childhood

Summary

Calcification of intervertebral discs is a rare

Recibido;18-08-05. Aceptado: 23-08-05 occurrence in children. Although the etiology of the calcification remains uncertain, it is no related with degenerative diseases. The clinical picture is non specific with neck pain, muscle contractures and, sometimes, low-grade fever. These symptoms generally disappear spontaneously, and surgery rarely becomes necessary. In our review of the literature, we have found only seventeen cases requiring surgical management. Two of these children had been previously diagnosed with calcified intervertebral discs, but at the time no herniation had been ruled out with magnetic resonance imaging (MRI). We report the case of a girl who was diagnosed, when she was ten years old, with intervertebral idiopatic calcifications. Four years later she presented with radiculopathy caused by the posterolateral displacement of a calcified cervical disc, wich required operative management. We think that this case supports the theory of Heinrich et al. that considers that the calcified hernia is a complication of a previous pathology, namely intervertebral calcification. Clinical and therapeutic aspects of this entity are discussed.

KEY WORDS: Anterior discectomy. Calcified herniated disc. Calcified intervertebral discs. Herniated disc in childhood.

\section{Introducción}

Las calcificaciones idiopáticas de los discos intervertebrales en la infancia (CIII), descritas por primera vez por Baron en $1924^{2}$, son una entidad infrecuente. Clínica e histológicamente constituyen un proceso diferente de las calcificaciones en los adultos: mientras en los niños las calcificaciones se dan en los núcleos pulposos, son más frecuentemente cervicales, sintomáticas y transitorias, en los adultos se localizan en el anillo fibroso, suelen ser torácicas y lumbares, asintomáticas y permanentes ${ }^{4,14,19}$. Estas calcificaciones han sido descritas en niños de todas las edades,

Abreviaturas. CIII: Calcificaciones idiopáticas de los discos intervertebrales en la infancia. 


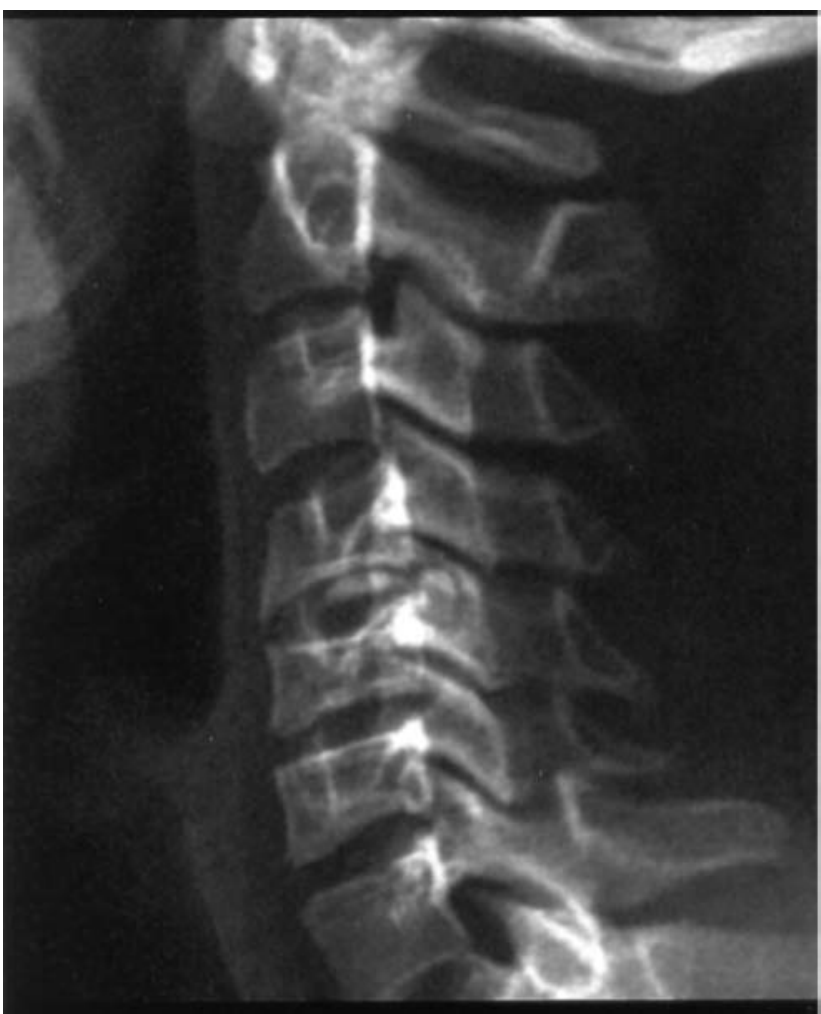

Figura 1. Rx cervical de 2003, en la que se aprecia pérdida de altura de C4-C5-C6 y una calcificación entre C4-C5. Puede observarse el desplazamiento posterior de la calcificación (comparar con la figura 4a).

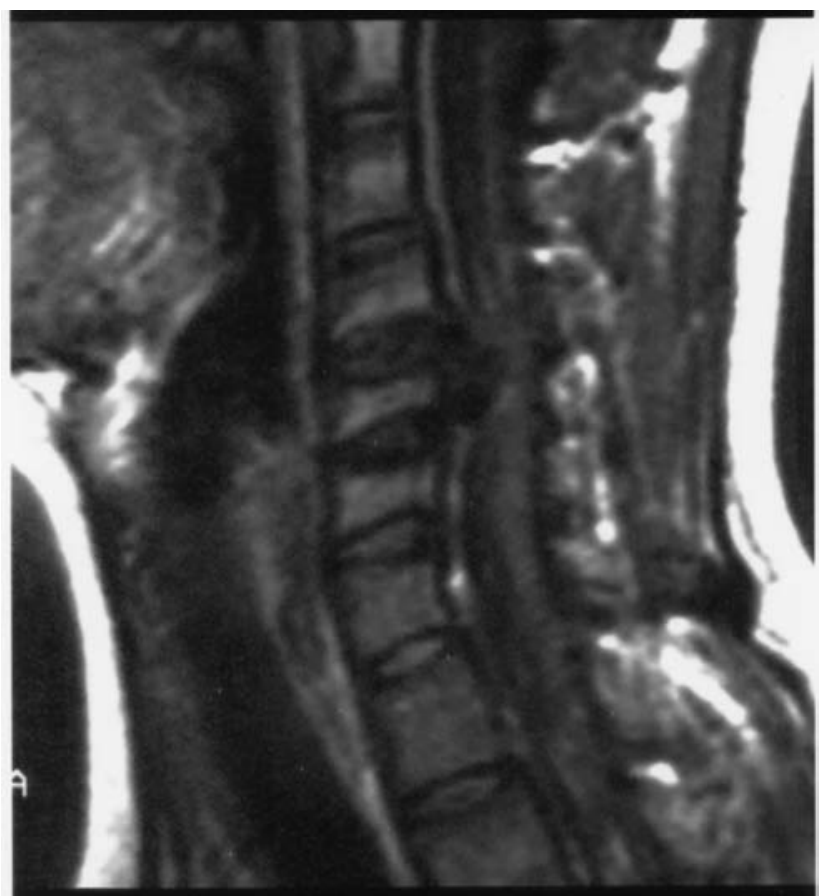

incluso en recién nacidos, con una media de edad de entre 5 y 8 años y ligero predominio en varones ${ }^{6,11,22}$. La columna cervical se afecta con mayor frecuencia, seguida de la torácica, siendo muy raros en la columna lumbar. En un $30-40 \%$ de los casos las calcificaciones son múltiples ${ }^{5}$. La etiopatogenia de la calcificación es todavía desconocida. Se barajan las posibilidades de un traumatismo, antecedente que se recoge en un $30-40 \%$ de los casos; de un proceso infeccioso, existiendo algunos discos intervenidos en los que se aprecia la existencia de células inflamatorias ${ }^{14,17,21} ; \mathrm{y}$, finalmente, la de un proceso isquémico ${ }^{3,11,22}$, llegando algunos autores a apuntar la teoría de una necrosis avascular del disco intervertebral y de las vértebras adyacentes que justificaría las alteraciones (geodas, pérdidas de altura y aplanamientos), que se observan frecuentemente en las vértebras próximas ${ }^{11}$. Las calcificaciones dorsolumbares, por su relativa inmovilidad, se suelen mantener asintomáticas y con escasa tendencia a la desaparición; las cervicales dan clínica más frecuentemente, generalmente de forma muy aguda, con intenso dolor cervical y contractura muscular. El inicio de la clínica suele coincidir con la fragmentación de la calcificación, que a su vez marca el inicio de su desaparición, probablemente por un proceso inflamatorio o autoinmune responsable de la reabsorción del material discal alterado ${ }^{4}$. Ocasionalmente un disco calcificado se hernia, y en estos casos puede aparecer clínica neurológica. Sin embargo, el pronóstico tampoco empeora sustancialmente, y es extremadamente raro que se requiera la intervención quirúrgica.

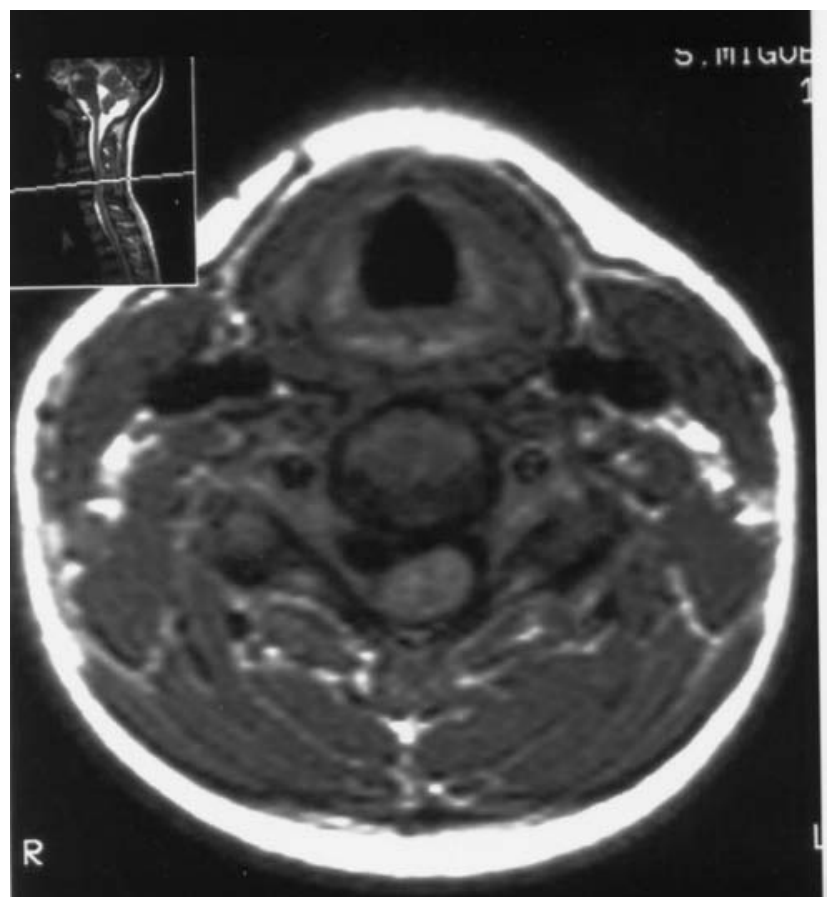

Figuras 2a y 2b. Cortes sagital y axial de RM cervical de 2003, en los que se aprecia una hernia discal entre C4-C5, que contacta con la raíz C5 derecha. Se aprecia también la disminución en altura de los cuerpos vertebrales, sin afectación de los discos. 


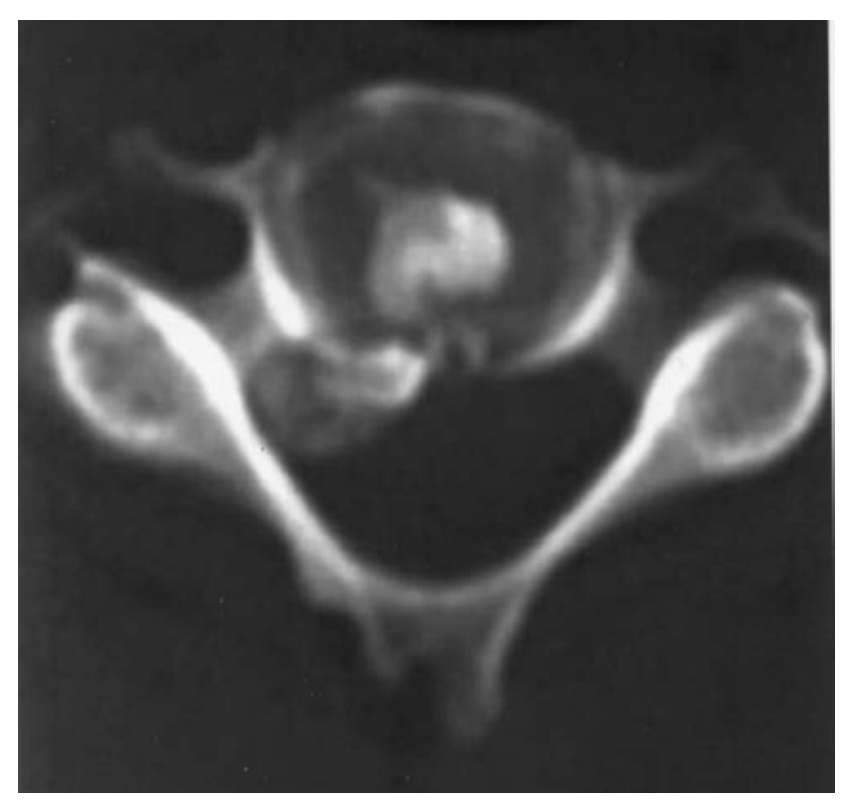

Figura 3. TC cervical en la que se aprecia la hernia con densidad calcio, así como la calcificación del disco.

En nuestra revisión de la literatura hemos encontrado solamente 17 casos de hernia discal calcificada en la infancia que requirieron tratamiento quirúrgico.

\section{Caso clínico}

Adolescente de 14 años, que ingresa para tratamiento de una hernia discal cervical a nivel C4-C5. Un mes antes había comenzado a sufrir cervicobraquialgia derecha y contracturas musculares cervicales, sin déficits

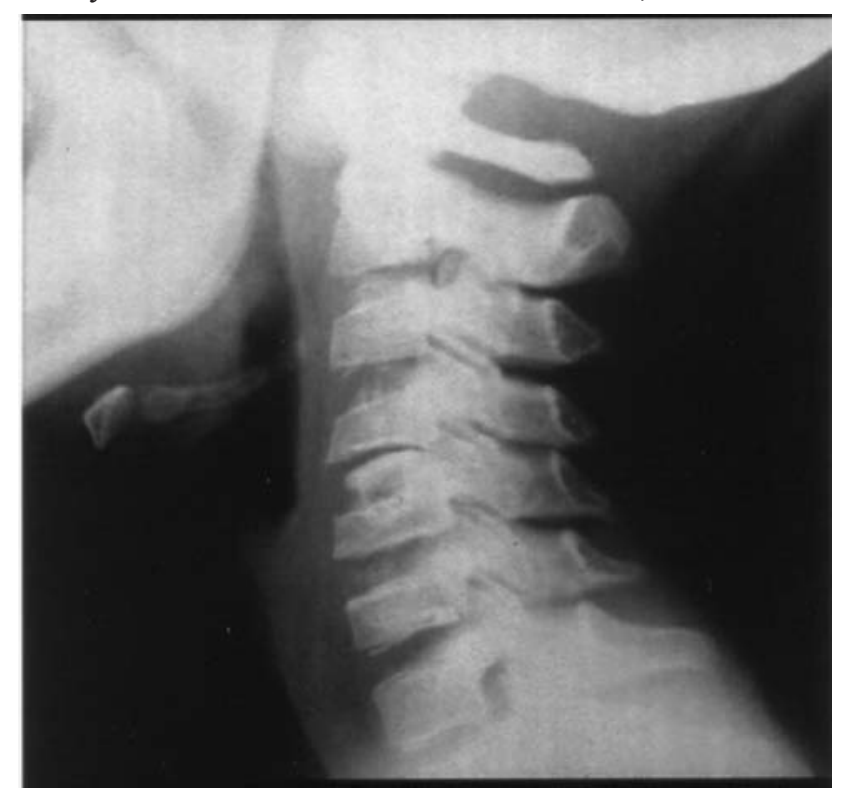

2006; 17: 333-339

neurológicos. El dolor mejoró con tratamiento conservador, pero días después comenzó con dificultad para la abducción del miembro superior derecho. En la exploración física destacaba una pérdida de fuerza en el deltoides y bíceps derechos (grados 2/5 y 4/5 respectivamente). No se apreciaron alteraciones sensitivas, ni piramidalismo en miembros inferiores. Las radiografías de columna cervical mostraban pérdida de altura en los cuerpos vertebrales de C4-C5-C6, así como una calcificación a nivel del disco C4-C5 (Figura 1). La RM cervical ponía de manifiesto una voluminosa hernia discal C4-C5 derecha que comprometía la raíz ipsilateral (Figuras 2a y 2b). Una tomografía computarizada (TC) cervical demostró que la hernia estaba calcificada (Figura 3). Se realizó un estudio analítico completo, no apreciándose alteraciones en el hemograma, en la velocidad de sedimentación globular (VSG), o en el metabolismo fosfocálcico. Una gammagrafía descartó captaciones patológicas.

Esta paciente había sido diagnosticada a la edad de 10 años de calcificaciones intervertebrales idiopáticas a raíz de un estudio radiográfico motivado por dolor cervical. Dicho estudio mostró la existencia de una calcificación a nivel de C4-C5 y varias calcificaciones a nivel dorsal (Figuras 4a y 4b). Una RM descartó cualquier herniación discal (Figuras 5 a y 5 b). Hasta el episodio actual había permanecido asintomática, salvo ocasionales dolores de espalda.

Dado el déficit neurológico que presentaba la paciente, se optó por el tratamiento quirúrgico de la hernia discal por

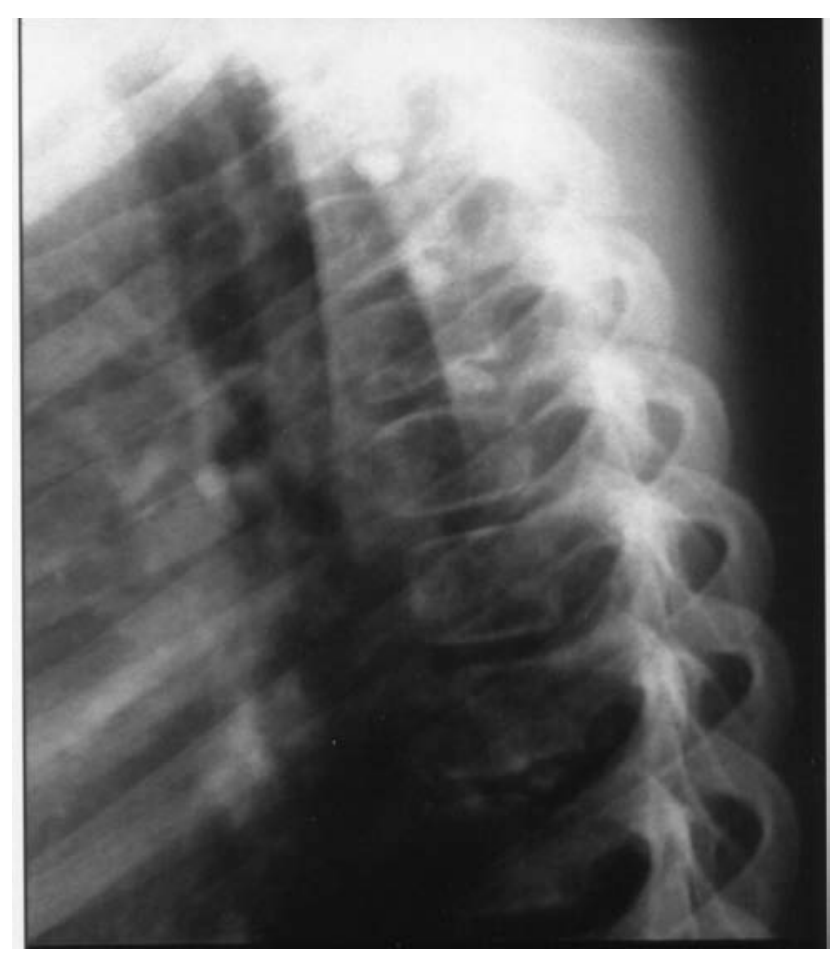

Figuras 4a y 4b. Rx de columna cervical y torácica de 1998 en las que se aprecia una calcificación entre C4-C5 y varias calcificaciones a nivel torácico. 


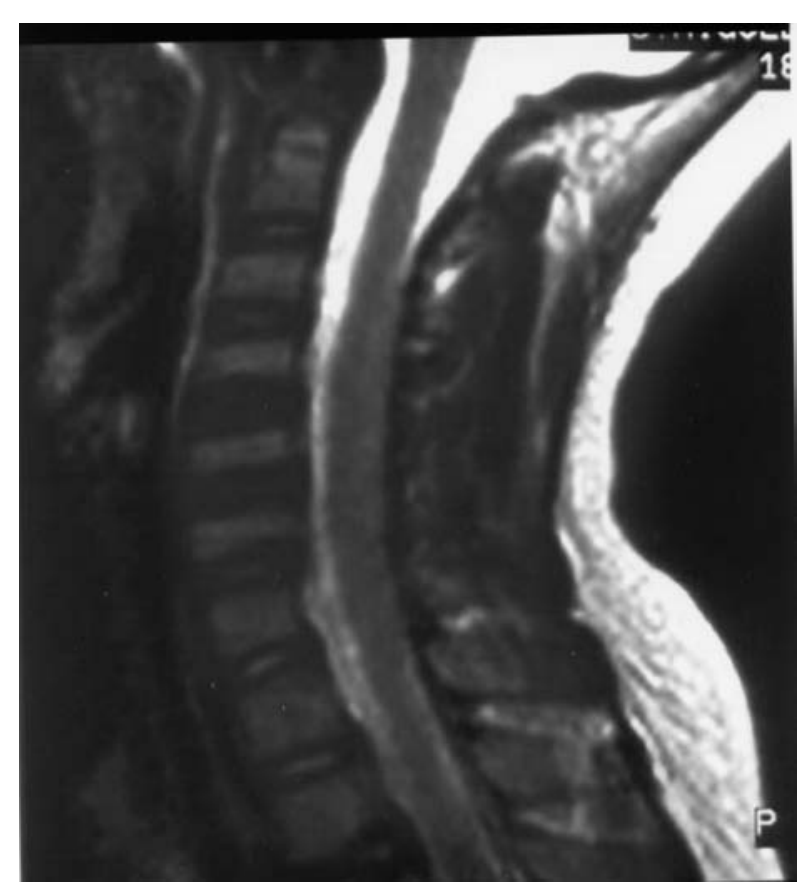

Figuras 5a y 5b. Cortes sagital y axial de RM de columna cervical de 1998 en la que se observa la ausencia de protrusión discal a nivel C4-C5.

vía anterolateral. Durante la cirugía se observó la existencia de un disco intervertebral con calcificaciones finas. Al abrir el ligamento vertebral común posterior se produjo la salida de un material blanquecino y pastoso, que se encontraba a moderada tensión. El informe patológico del material obtenido fue de fibrocartílago con focos de calcificación. Un mes después la paciente se había recuperado por completo de sus déficits.

\section{Discusión}

La presencia de hernias discales en los niños es un hecho infrecuente, y más raro aún es que estén calcificadas y se acompañen de clínica neurológica. Algunos autores han sugerido que un disco calcificado herniado puede representar la evolución de una hernia no diagnosticada previamente y que posteriormente ha sufrido un proceso de calcificación ${ }^{1}$. Sin embargo, la mayoría acepta la teoría propuesta por Heinrich y cols., que consideran que la calcificación en el disco intervertebral es un primer paso para la protrusión sintomática del mismo, debido al cambio que el calcio origina en las características mecánicas y anatómicas del complejo discal ${ }^{8}$. Según Sonnabend ${ }^{22}$, hasta en un $38 \%$ de los pacientes con calcificaciones en los discos intervertebrales que tienen síntomas se puede demostrar una protrusión radiológica de la calcificación, cosa que no ocurre en los pacientes asintomáticos. Sin embargo, la clínica se limita a síntomas raquídeos. La aparición de disfagia o síntomas radiculares o de compresión medular es

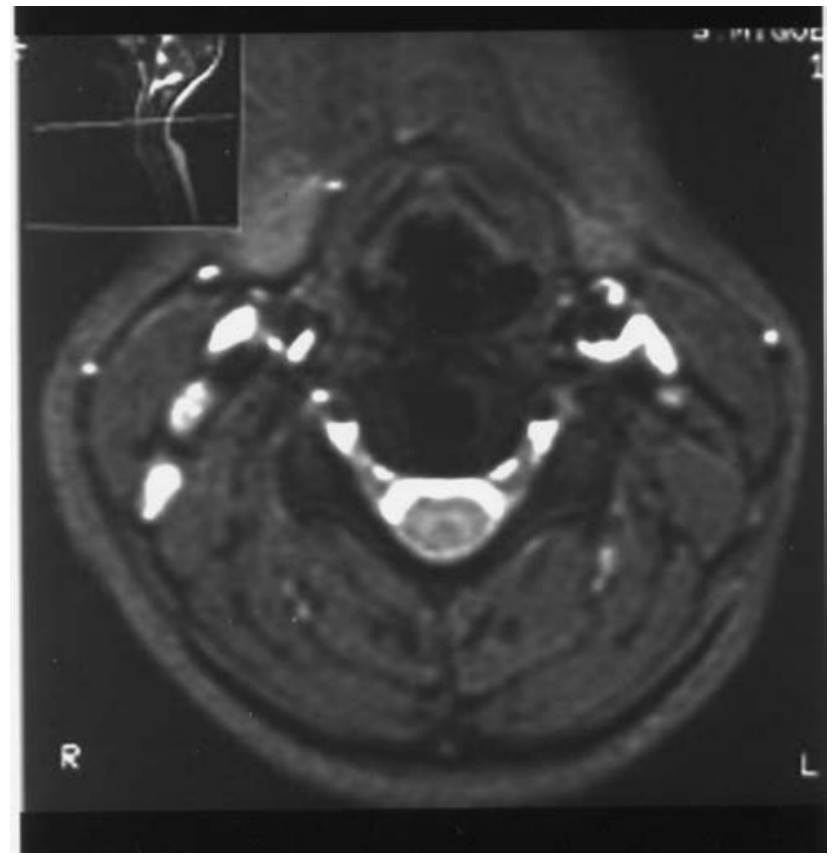

excepcional y se aprecia sólo en un 5-6\% de estos pacientes sintomáticos. A pesar de todo, tan solo dos de los pacientes que han requerido tratamiento quirúrgico por una hernia calcificada habían sido diagnosticados previamente de CIII, mediante radiografía cervical ${ }^{10,17}$. Hasta ahora no se había conseguido documentar el paso de CIII a hernia mediante RM espinal. El caso presentado por nosotros apoya definitivamente dicha teoría, ya que aportamos no sólo estudio radiográfico que confirma las calcificaciones discales 4 años antes, sino también RM en la que se observa la inexistencia de hernia discal previa.

La calcificación intervertebral asintomática no precisa tratamiento. Cuando la calcificación es sintomática sólo requiere tratamiento conservador con analgesia, reposo y relajantes musculares. La tendencia de la misma es, una vez iniciada la clínica, hacia su desaparición, y lo mismo ocurre en el caso de la hernia calcificada que no produce clínica neurológica ${ }^{4,22}$. El problema lo plantea la hernia calcificada que ha producido clínica neurológica o disfagia. En estos casos, y a diferencia de lo que ocurre con las hernias blandas de la infancia, en las que se recomienda el tratamiento quirúrgico precoz si se han producido déficits neurológicos ${ }^{7}$, la mayoría de los autores aconsejan el tratamiento conservador, precisamente porque la tendencia de estas hernias es hacia la desaparición. La extirpación quirúrgica de las mismas queda reservada para casos con dolor radicular intratable, disfagia o déficits neurológicos persistentes y significativos que no cedan a tratamiento conservador $^{4,21}$. 
Tabla I

Casos recogidos en la literatura de hernia calcificada idiopática en menores de 16 años que han requerido tratamiento quirúrgico. Se excluyen los casos asociados a patología conocida (raquitismo, hipervitaminosis D, ocronosis, etc)

\begin{tabular}{|c|c|c|c|c|c|}
\hline Autor & Sexo/Edad & Nivel & Clínica & Tratamiento & Recuperación \\
\hline Peck Jr $1957^{18}$ & $\mathrm{~V}, 12$ & T6-T7 & Mielopatía & Laminectomía con curetaje & Completa \\
\hline $\begin{array}{l}\text { McCartee Jr, } \\
\text { y col. } 1972^{13}\end{array}$ & $\mathrm{M}, 12$ & T12-L1 & Mielopatía & Laminectomía & Completa \\
\hline Swick $1975^{23}$ & $\mathrm{M}, 10$ & T4-T5 & Mielopatía & Laminectomía & $\begin{array}{l}\text { Completa (cifosis } \\
\text { postlaminectomía) }\end{array}$ \\
\hline Swick. $1975^{23}$ & $\mathrm{~V}, 11$ & $\mathrm{C} 6-\mathrm{C} 7$ & Radiculopatía & Discectomía & Completa \\
\hline Smith y col. $1977^{21}$ & $\mathrm{~V}, 12$ & C4-05 & Radiculopatía & Discectomía & Completa \\
\hline $\begin{array}{l}\text { Reale y Gambacorta } \\
\qquad 1985^{19}\end{array}$ & $\mathrm{~V}, 16$ & L4-L5 & Radiculopatía & Laminectomía con discectomía & Completa \\
\hline Nicolau y col. $1985^{16}$ & $\mathrm{~V}, 10$ & C6-C7 & Radiculopatía & Discectomía & Completa \\
\hline Hoeffel y col. $1990^{9}$ & $\mathrm{~V}, 12$ & C6-C7 & Disfagia & Discectomía & Completa \\
\hline Mohanty y col.1992 ${ }^{15}$ & $\mathrm{M}, 8$ & $\mathrm{C} 6-\mathrm{C} 7$ & Radiculopatía & Discectomía & Completa \\
\hline Mohanty y col.1992 15 & M, 16 & C6-C7 & Mielopatía & Discectomía & Completa \\
\hline Oga y col $1993^{17}$ & $\mathrm{~V}, 12$ & C7-T1 & $\begin{array}{l}\text { Radiculopatía, } \\
\text { mielopatía }\end{array}$ & Discectomía & $\begin{array}{l}\text { Recuperación } \\
\text { motora } 4 / 5\end{array}$ \\
\hline Hoffman y col. $1998^{10}$ & M, 11 & C7-T1 & Radiculopatía & Hemilaminectomía & Completa \\
\hline Knupfer y col. $2000^{12}$ & $\mathrm{M}, 11$ & C7-T1 & $\begin{array}{l}\text { Radiculopatía, } \\
\text { mielopatía }\end{array}$ & Discectomía & Completa \\
\hline Gerlach y col. $2001^{5}$ & M, 10 & $\mathrm{C} 7-\mathrm{T} 1$ & Mielopatía & Discectomía & Completa \\
\hline Mahlfeld y col. $2002^{14}$ & $\mathrm{M}, 11$ & $\mathrm{~T} 2-\mathrm{T} 3$ & Mielopatía & $\begin{array}{l}\text { Laminectomía con } \\
\text { discectomía }\end{array}$ & $\begin{array}{l}\text { Persiste déficit } \\
\text { motor y sensitivo } \\
\text { en MII }\end{array}$ \\
\hline Aulisa y col. $2003^{1}$ & M, 16 & L5-S1 & Mielopatía & Descompresión raíz $\mathrm{S} 1$ & Completa \\
\hline Schaser y col. $2003^{20}$ & $\mathrm{~V}, 12$ & $\mathrm{C} 3-\mathrm{C} 4$ & Mielopatía & Discectomía & Completa \\
\hline Ortega y col. 2004 & M, 14 & $\mathrm{C} 4-\mathrm{C} 5$ & Radiculopatía & Discectomía & Completa \\
\hline
\end{tabular}

MII: miembro inferior izquierdo

En nuestra revisión de la literatura hemos encontrado tan sólo 17 casos que hayan sido tratados quirúrgicamente (Tabla I). Aunque en la mayoría de los casos la recuperación fue completa, en dos ocasiones persistieron déficits neurológicos ${ }^{14,17}$, lo cual podría justificar en algunos casos, especialmente aquellos con afectación medular, una intervención quirúrgica más precoz. Nuestra paciente, diagnosticada a la edad de 10 años de CIII, presentó 4 años después un cuadro de radiculopatía con déficit neurológico establecido, debido a herniación posterolateral de uno de los discos calcificados. Dado el déficit neurológico que presentaba, se optó por el tratamiento quirúrgico, realizándose 
discectomía cervical anterior y fusión de cuerpos vertebrales, con una excelente evolución posterior.

\section{Conclusión}

Las CIII son un cuadro raro, benigno y habitualmente autolimitado. Generalmente son asintomáticas y cuando dan clínica, ésta suele limitarse a síntomas raquídeos y responden a tratamiento conservador. Excepcionalmente, un disco calcificado preexistente se complica con herniación anterior o posterior del fragmento calcificado, originando déficits neurológicos o disfagia. En estos casos, cuando la clínica no cede ante el tratamiento conservador, está indicada la intervención quirúrgica, que casi siempre va seguida de una excelente recuperación de los déficits.

\section{Bibliografía}

1. Aulisa, L., Pitta, L., Aulisa, A.G., Mastantuoni, G., Pola, E., Leone, A.: Lumbar nerve root walled by a calcified herniated mass in a young patient. Childs Nery Syst. 2003; 19: 384-386.

2. Baron, A.: Uber eine neue Erkrankung der Wirbelsaüle. Jahrb. Kinderh. 1924; 104: 357-360.

3. Bollini, G., Bergoin, M., Choux, M., Padovani, J.: Calcifications discales de 1'enfant. A propos de 17 cas. Revue de Chirurgie Orthopédique. 1984; 70: 377-382.

4. Dias, M. S., Pang, D.: Juvenile intervertebral disc calcification: recognition, management, and pathogenesis. Neurosurgery. 1991; 28: 130-135.

5. Gerlach, R., Zimmermann, M., Kellermann, S., Lietz, R., Raabe, A., Seifert, V.: Intervertebral disc calcification in childhood. A case report and review of the literature. Acta Neurochir. 2001; 143: 89-93.

6. Girodias, J.B., Azouz, E.M., Marton, D.: Intervertebral disk space calcification. A report of 51 children with a review of the literature. Pediatr Radiol. 1991; 21: 541-546.

7. Goumnerova, L.C., Adelson, P.D.: Diseases of the intervertebral disc in children. En Youmans (ed). Neurological Surgery. Philadelphia.; W.B. Saunders Company, 1996; pp 2375-2381.

8. Heinrich, S.D., Zembo, M.M., King, A.G., Zerkle, A.J., MacEwen, G.D.: Calcific cervical intervertebral disc herniation in children. Spine. 1991; 16: 228-231.

9. Hoeffel, J.C., Bernard, C., Schmit, P., Denardaud, L.: Herniation of calcifying discopathy in childhood: report of three cases. Eur J Pediatr. 1990; 149: 695-697.

10. Hoffman, A.I., Lambiase, R.E., Levine, S.M.: Intervertebral cervical disk calcification requiring operative management in a child. AJR. 1998; 171: 898-899.
11. Jawish, R., Rigault, P., Padovani, J.P., Mouterde, P., Thouzet, Ph., Chaumien, J.P.: Calcifications discales intervertébrales chez 1'enfant. Revue de Chirurgie Orthopédique. 1989; 75: 308-317.

12. Knüpfer, M., Rieske, K., Pulzer, F., Trantakis, C., Dietrich, J., Handrick, W.: Prolaps einer verkalkten Bandscheibe als Ursache eines drohenden Querschnittssyndroms bei einem jungen Mädchen. Klin Pädiatr. 2000; 212: 117-120.

13. Maccartee, C.C., Griffin, P.P., Byrd, E.B.: Ruptured calcified thoracic disc in a child: report of a case. J Bone Joint Surg. 1972; 54: 1272-1274.

14. Mahlfeld, K., Kayser, R., Grabhoff ,H.: Permanent thoracic myelopathy resulting from herniation of a calcified intervertebral disc in a child. J Pediatr Orthop. 2002; 11: 6-9.

15. Mohanty, S., Sutter, B., Mokry, M., Ascher, P.W.: Herniation of a calcified cervical intervertebral disk in children. Surg Neurol. 1992; 38: 407-410.

16. Nicolau, A., Diard, F., Darrigade, J.M., Dorcier, F., Vital, J.M.: Hernie postérieure d'un disque calcifié chez 1 'enfant. A propos de 2 observations. J Radiol. 1985; 66: 683688 .

17. Oga, M., Terada, K., Kikuchi, N., Oda ,Y., Sugioka, Y.: Herniation of calcified cervical intervertebral disc causes dissociated motor loss in a child. Spine. 1993; 18: 2347-2350.

18. Peck, F.C., Jr.: A calcified thoracic intervertebral disk with herniation and spinal cord compression in a child: case report. J Neurosurg. 1957; 14: 105-109.

19. Reale, F., Gambacorta, D.: Calcified lumbar disc protrusion in an adolescent. Surg Neurol. 1985; 24: 661-662.

20. Schaser, K.D., Stover, J.F., Kaeaeb, M.J., Haas, N.P., Mittlmeier, T.: Mild cervical spine trauma showing symptomatic calcified cervical disc herniation in a child: a case report. Spine. 2003; 28: E93-E94.

21. Smith, R.A., Vohman, D., Dimon, J.H., Averett, J.E. Jr., Milsap, J.H. Jr.: Calcified cervical intervertebral discs in children: report of three cases. J Neurosurg. 1977; 46: 233-238.

22. Sonnabend, D.H., Taylor, T.K.F., Chapman, G.K.: Intervertebral disc calcification syndromes in children. . J Bone Joint Surg. 1982; 64: 27-31.

23. Swick,, H.M.: Calcification of intervertebral discs in childhood. J Pediatr. 1975; 86: 364-369.

Ortega-Martínez, M.; Cabezudo, J.M.; Fernández-Portales, I.; Gómez-Perals, L.; Bernal-García. L.M.: Hernias discales calcificadas en la infancia. Neurocirugía 2006; 17: 333-339.

Correspondencia postal: Dra. Marta Ortega Martínez. Servicio de Neurocirugía. Hospital Infanta Cristina. Avda de Elvas s/n. Badajoz. 
Comentario al trabajo: Hernias discales calcificadas en la infancia de Ortega-Martínez y cols.

La calcificación del disco intervertebral en el niño es poco frecuente. Su incidencia es desconocida ya que los pacientes pueden permanecer asintomáticos. A veces las calcificaciones son encontradas durante el estudio de un niño o adolescente con dolor de cuello o espalda. Las calcificaciones del disco son más habituales en la columna dorsal, seguidas de las de la región cervical y lumbar. El cuadro clínico típico consiste en (1) pacientes en edad pediátrica, (2) con dolor local o referido, (3) contractura espinal o tortícolis, (4) frecuentemente con indicios de inflamación, (5) calcificación visible en los estudios radiográficos, (6) curso autolimitado, (7) buena respuesta al tratamiento conservador. El diagnóstico, sobre todo desde el punto de vista académico, incluye: (1) traumatismos, (2) inflamación discal, (3) hipervitaminosis D, (4) alteraciones diversas del metabolismo calcio/fósforo y (5) alcaptonuria. En ésta última enfermedad las calcificaciones suelen ser múltiples. El origen de las calcificaciones discales está íntimamente relacionado con el de la hernia discal infantil y las discitis ${ }^{1,2}$.

El caso que se describe es muy interesante porque ilustra la posibilidad de recidiva de la calcificación discal que, finalmente, se hizo sintomática. Aunque el tratamiento clásico de la calcificación discal es conservador, algunos casos -como el aquí descrito- evolucionan con signos y síntomas de compresión radicular o medular y requieren tratamiento quirúrgico. La información suministrada en el trabajo, y en la Tabla, sobre esta entidad es exhaustiva y supone una completa actualización de un tema tan infrecuente e interesante.

\section{Bibliografía}

1. Martínez-Lage, J.F., López Hernández, F.: Hernia discal en niños y adolescentes. En Villarejo FJ, Martínez-Lage JF (eds) Neurocirugía Pediátrica. Madrid; Ergon, 2001; pp. 51330

2. Matson, D.D.: Neurosurgery of infancy and childhood. Springfield (Ill.); Thomas, 1969, pp. 376-380 y 737

J.F. Martínez-Lage Murcia 